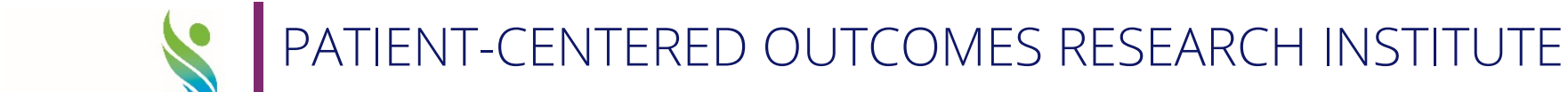 pcori). RESEARCH SUMMARY
}

\section{Assessing Personalized Care Plans for Patients Who Received Hematopoietic Cell Transplant for Cancer Treatment}

Principal investigator

Elizabeth Murphy, MS, EdD
Organization

National Marrow Donor Program

\section{What was the research about?}

Hematopoietic cell transplant, or HCT, also called bone marrow transplant or stem cell transplant, is a type of cancer treatment. This procedure replaces damaged cells in patients whose normal blood cells have been affected by cancer. People who have no signs of cancer after finishing treatment are often called HCT survivors. HCT survivors may need follow-up care for years to prevent future health problems.

In this study, the research team created and tested a personalized survivorship care plan, or SCP, for HCT survivors. An SCP is a document that describes survivors' cancer diagnoses and treatments. It also lists check-ups and tests that survivors need after treatment, questions to ask their doctors, and steps for improving health.

The research team compared HCT survivors who received an SCP with those who received usual posttransplant care without an SCP.

\section{What were the results?}

Having an SCP didn't increase HCT survivors' confidence in understanding their cancer, treatment, and follow-up care more than usual care. General health, quality of life, and use of health care also didn't differ between the two groups.

HCT survivors who received an SCP had less distress related to their cancer treatment and better mental health-related quality of life compared with those who received usual care.

\section{Who was in the study?}

The study included 495 HCT survivors who had HCT one to five years ago, whose cancer hadn't returned, and who hadn't developed a new cancer. Of these, 94\% were white, $4 \%$ were African American, 3\% were Hispanic, and $1 \%$ were Asian. The median age was 59, and $54 \%$ were men.

\section{What did the research team do?}

The research team assigned HCT survivors to one of two groups by chance. The first group received a personalized SCP. SCPs were personalized for each patient using information about other patients like them who also had HCTs. The second group received usual follow-up care. Patients in both groups completed surveys by phone before the study started and again six months later.

Patients, caregivers, doctors, and social workers helped the research team design the SCP.

\section{What were the limits of the study?}

The SCPs didn't include details about treatments HCT survivors had before their transplants. These details may affect survivors' long-term risks. People in the study had HCT one to five years ago; most were white. Results may differ for people from other backgrounds.

Future research could test different SCP formats and other ways to increase patients' confidence in survivorship care. Studies could also include patients from other backgrounds. 


\section{How can people use the results?}

Researchers and others working to support HCT

survivors can consider these results when looking at ways to help HCT survivors better understand their long-term follow-up health care.

To learn more about this project, visit www.pcori.org/Murphy193. 